\title{
The optical mission behind the Stratos Project (Presentation Video)
}

\section{Dennis Fisher}

Dennis Fisher, "The optical mission behind the Stratos Project (Presentation Video)," Proc. SPIE 8842, Novel Optical Systems Design and Optimization XVI, 88420C (18 November 2013); doi: 10.1117/12.2030235

EDIE Event: SPIE Optical Engineering + Applications, 2013, San Diego, California, United States 


\title{
Introduction to the Stratos Project: The Optical Mission Behind the Stratos Project
}

\section{(Presentation Video)}

Joseph B. Houston, Houston Research Associates (United States)

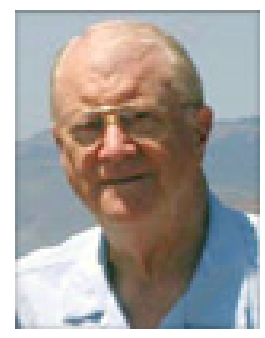

Dennis Fisher, Genesis Applied Imaging, Inc. (United States)

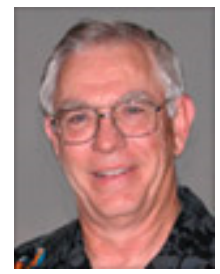

\begin{abstract}
The recent Red Bull Stratos Project enabled Felix Baumgartner to skydive from 127,852 feet, breaking a number of world records, and also become the first person to break the sound barrier in free fall. This incredible example of human achievement was documented by a group of optical imaging scientists who worked for over four years to develop and test the necessary systems and equipment. This monumental leap was captured by 35 cameras to provide a full picture of what this endeavor means for the future of scientific exploration. This special presentation will feature an insider look behind the Stratos Project: goals, challenges, and lessons learned.
\end{abstract}

View presentation video on SPIE's Digital Library: http://dx.doi.org/10.1117/12.2030382 